\title{
The Functional Head Impulse Test and Vestibular Rehabilitation with V-Gym: A Diagnostic and Therapeutic System for the Semicircular Canals
}

\author{
Anirban Biswas ${ }^{1}$ \\ ${ }^{1}$ Vertigo and Deafness Clinic, Kolkata, West Bengal, India
}

Ann Otol Neurotol ISO 2018;1:49-50

\section{Introduction}

The functional status of the semicircular canals can be tested by the caloric test video nystagmography/electronystagmography (VNG/ENG), dynamic visual acuity (DVA) test, gaze stabilization test (GST), and video head impulse test (VHIT). All these tests have certain limitations, for example the caloric test ENG/VNG as well as the DVA and GST evaluates only the lateral canals, and it is only the VHIT that evaluates all the six canals with precision. However, one common disadvantage is that all these tests are pretty cumbersome and involve very costly and sophisticated instrumentation that most clinicians cannot afford at least in the private sector. This new test called the functional head impulse test (fHIT) is a new addition to the neurotologist's diagnostic armamentarium for evaluation of all the six semicircular canal that addresses many of the limitations of the other available tests. The fHIT reliably measures the functional status of all the six semicircular canals by ascertaining the efficiency of the rotational vestibulo-ocular reflex (rVOR) in a quick, very simple, easy-to-perform way and with a perfectly physiologic stimulus. The fHIT precisely assesses whether the rVOR of the semicircular canals are being able to stabilize images when there is a fast head movement in the plane of the semicircular canals. After a defective canal has been detected, the system has inbuilt rehabilitative methods that can stimulate the canal and enhance its functionality. In addition to its simplicity of design and affordable costs (without compromising reliability), the fHIT system has the unique advantage of being a compact diagnostic-cum-therapeutic device and is a boon to practioners in neurotology (-Fig. 1).

\section{Relevance of Functional Head Impulse Test in Modern Vestibulometry}

Functional head impulse test does not ascertain the vestibulo-ocular reflex (VOR) gain that the VHIT does or measure the percentage of canal paresis that the caloric test ENG/VNG does, or even measure the loss of visual acuity that the DVA test does, but it assesses whether the semicircular canals are
Address for correspondence Anirban Biswas, Vertigo and Deafness Clinic, BJ-252, Salt Lake, Sector-2, Kolkata 700091, India (e-mail: vertigodeafnesstinnitus@gmail.com).

being able to serve the function of gaze stabilization and, if so, how efficiently. This qualitative rather than quantitative measurement is a more practical approach and more relevant to the clinician as all that the clinical practitioner in neurotology is interested to know is whether the semicircular canals are serving their function of maintaining a stable gaze when there is a fast head movement. If a stable gaze is not maintained during a fast head movement in the plane of a particular semicircular canal then that canal is considered to be malfunctioning. The fHIT can detect this very well by ascertaining loss of readability during fast head movements.

\section{Vestibulo-ocular Reflex, Visual Acuity, and Functional Head Impulse Test}

The VOR keeps the visual objects in focus and maintains a stable gaze with perfect visual acuity during fast head movement. The semicircular canals sense the head movement very accurately, and the oculomotor centers then generate the requisite eye movement reflexly to ensure gaze stabilization.

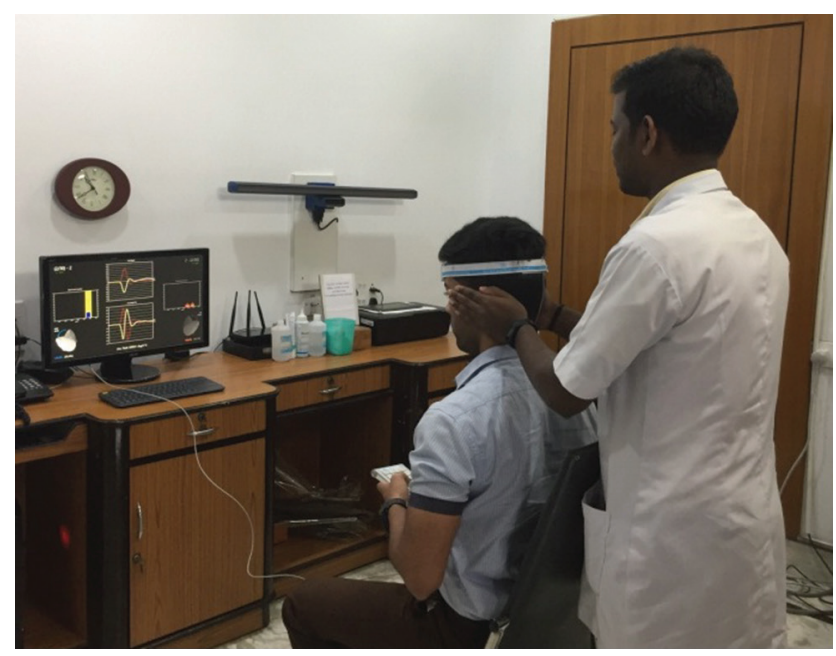

Fig. 1 The functional head impulse test being carried out at the author's clinic. 

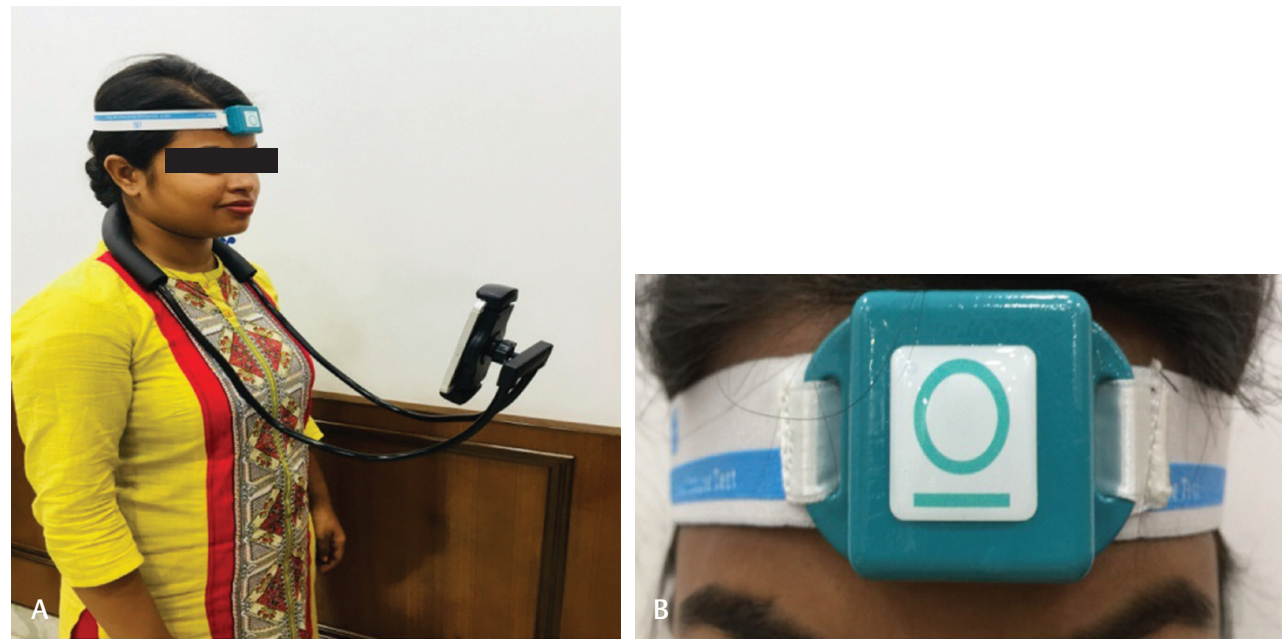

Fig. 2 (A) The VGym rehabilitation being carried out using the patient's mobile phone with the requisite rehabilitation app installed in it. The patient can walk around while performing the rehabilitation exercise. (B) The hardware for the VGym rehabilitation consists of just the headband with the gyroscope fitted on it.

The reflex mechanism is known as the VOR, and as the semicircular canals detect only rotational acceleration, that is, acceleration when the head is rotated at any angle, this VOR is called rotational VOR (rVOR).

Visual acuity is the clarity of vision and is dependent on the stability and sharpness of the retinal focus within the eye. Readability or the faculty of recognizing a visual object like a letter/ Figure / picture depends on visual acuity. If the images of the visual surroundings are not stable in the retina, there will naturally be a loss of visual acuity. If a semicircular canal is defective, then the rVOR which is generated from the inputs from that semicircular canal is poor and consequently images of the visual surroundings slip in the retina (loss of gaze stabilization) whenever there is a sudden fast head movement in the plane of that semicircular canal. This causes a loss of the sharpness of the visual focus which in turn leads to a blurring of vision and a loss of visual acuity and consequently a degradation of readability. The functional Head Impulse Test (fHIT) ascertains if there is degradation or impairment of readability on sudden head movement and thereby identifies any impairment of the rVOR generated from that semicircular canal in the plane of which the head movement is taking place. It not only detects the defective semicircular canal but also detects at which range of accelerations is the semicircular canal malfunctioning. The fHIT informs the neurotologist that (say) the left posterior semicircular canal is malfunctioning at accelerations between 5,000 to 6,000 degrees/ $/ \sec ^{2}$ but is perfect at accelerations between 2000 to 5000 degrees $/ \mathrm{sec}^{2}$. This is the precision of the fHIT, and it does so for all the six semi-circular canals in a few minutes in a simple, nonexpensive but very reliable way.

\section{The Vestibular Rehabilitation with V-Gym}

Modern vestibular physiotherapy entails specific organtargeted exercises for specific disorders after modern vestibulometry has very precisely diagnosed the disorder and localized the site of lesion. The diagnostic and therapeutic parts of the fHIT fit into this model of modern neurotology that entails very accurate diagnosis and very precise and specific organ- or disease-targeted therapy. With fHIT, we can diagnose which particular semicircular canal is malfunctioning at which particular range of frequencies and then institute rehabilitative physiotherapy by V-Gym to stimulate that particular canal only at that particular acceleration (-Fig. 2).

The V-Gym consists of a gyroscope and is connected to the patient's mobile phone via Bluetooth. There are various programs of exercises to stimulate a particular semicircular canal for rehabilitative purposes at different speeds and at different levels of difficulties inbuilt in the fHIT and V-Gym systems. The rehabilitative programs are tailor-made to work according to the disease identified by fHIT test. Hence, a combination of fHIT and V-Gym provides a very easy and perfect solution for any vertigo management if the defect is in one or more of the semicircular canals. However, it does not test the otolith organs.

\section{Conclusion}

The fHIT as it stands today is NOT a replacement of other vestibulometric tests such as VNG, VHIT, DVA, GST etc. but a very useful addition to the neurotologist's diagnostic armamentarium and a boon to the practicing neurotologist in a big way. Possibly in the very near future it will even replace some of the other very costly and cumbersome tests. The USP is its simplicity, affordability, portability, and the negligible foot print. A gyroscope, which is the heart of the instrument is a very cheap widely available device that is present in all smart phones. We have been using the fHIT with its built-in rehabilitation system in our clinic for the past 2 months, and it has been a fascinating experience. This new device is a godsend to neurotologists because of its very simple mechanism that practically does not ever go out of order due to its extremely simple technology, its affordability, and, of course, its very precise diagnostic and therapeutic functions. For further reading, refer to the blog page of vertigoclinic.in. 